\title{
Significados de ser feliz na velhice e qualidade de vida percebida segundo idosos brasileiros
}

\author{
Meanings of "being happy in old age" and perceived quality of life according to Brazilian older adults
}

Catherine Nicol Aravena Valero' (ID

Tulia Fernanda Garcia Meira' $\mathbb{D}$ Daniela de Assumpçãol $\mathbb{D}^{1}$ Anita Liberalesso Neri',2 $\mathbb{1 D}$

\section{Resumo}

Objetivo: Identificar associações entre significados atribuídos ao conceito "ser feliz na velhice" e qualidade de vida percebida em amostra de idosos recrutados na comunidade. Métodos: Os dados foram extraídos dos bancos da linha de base (LB; 2008-2009) e do seguimento (SG; 2016-2017) do Estudo Fibra Campinas, de base populacional, sobre fragilidade em idosos. Participaram 211 indivíduos com idade média de 81,0 44,3 anos no SG, com registro de respostas a uma questão aberta sobre felicidade na velhice na LB e à escala CASP-19 de qualidade de vida percebida (SG). Os registros da LB foram submetidos a análise de conteúdo; os do SG a medidas de frequência, posição e dispersão, e ambos a análises de regressão logística. Resultados: Da análise de conteúdo foram derivados quatro temas: saúde e funcionalidade (o mais mencionado), bem-estar psicológico, relações interpessoais e recursos materiais. As categorias mais citadas foram autodesenvolvimento e relações familiares. Prevaleceram altas as pontuações na CASP19 , que foram mais prováveis entre os idosos que não mencionaram recursos materiais $(\mathrm{OR}=2,44$; IC95\%:1,20-4,43), nem saúde e funcionalidade (OR=2,03; IC95\%:1,22-4,22), e entre os que citaram relações interpessoais (OR=1,92; IC95\%:1,08-3,41) na LB. Altas pontuações no fator Autorrealização/Prazer foram mais prováveis entre os de 80-84 anos $(\mathrm{OR}=1,93$; IC95\%:1,01-3,68) e entre os que não citaram saúde e funcionalidade $(\mathrm{OR}=1,98$; IC95\%:1,00-1,98) na LB. Conclusão: Felicidade na velhice e qualidade de vida referenciada a necessidades psicológicas são construtos relacionados. Sua avaliação capta vivências que excedem condições materiais e de saúde, predominantes nas medidas clássicas de qualidade de vida na velhice.

\footnotetext{
Universidade Estadual de Campinas, Faculdade de Ciências Médicas, Programa de Pós-Graduação em Gerontologia. Campinas, SP, Brasil.

2 Universidade Estadual de Campinas, Faculdade de Ciências Médicas, Departamento de Psicologia Médica e Psiquiatria. Campinas, SP, Brasil.
}

Financiamento da pesquisa: Convênio CAPES/Procad 2972/14. Projeto No 88881.068447/14. Bolsa CAPES para Catherine Nicol Aravena Valero, No 02-P-4261/2020.

Os autores declaram não haver conflito na concepção deste trabalho.

Palavras-chave: Felicidade. Envelhecimento Saudável. Longevidade. Qualidade de Vida. 


\section{Abstract}

Objective: Identifyting associations between meanings of "being happy in old age" and perceived quality of life in a sample of community-dwelling older adults. Methods: Data were drawn from the baseline (BL; 2008-2009) and follow up (FW; 2016-2017) records of the Fibra Campinas, a population-based study on frailty in old age. Two hundred and eleven individuals with an average age of $81.0 \pm 4.3$ at follow-up, answered to an open question on happiness in old age at baseline and to the CASP-19 at follow-up. BL records were submitted to content analysis; those of FW to measures of frequency, position and dispersion, and both to logistic regression analyzes. Results: The content analysis generated four themes: health and functionality (the most mentioned), psychological well-being, interpersonal relationships and material resources. The most cited categories were self-development and family relationships. High scores in CASP-19 prevailed and were more likely among participants who did not mention material resources $(\mathrm{OR}=2.44$; 95\%CI: 1.20-4.43), nor health and functionality (OR=2.03; 95\% CI: 1.22-4.22), and among those who cited interpersonal relationships (OR=1.92; 95\%CI: 1.08-3.41) at BL. High scores in the factor Self-Realization/Pleasure were more likely among those aged 80-84 (OR=1.93; 95\%CI: 1.01-3.68) and among those who did not mention health and functionality (OR=1.98; 95\%CI: 1,00-1.98) at BL. Conclusion: Happiness in old age and quality of life related to psychological needs of control, autonomy, self-realization and pleasure are related constructs, whose evaluation captures experiences that exceed material and health conditions, predominant in classic measures of quality of life.

\section{INTRODUÇÃO}

Felicidade é um construto multidimensional, identificado com condições objetivas tais como saúde, independência, recursos materiais, apoio familiar e relações sociais, e com condições subjetivas tais como satisfação com a vida, senso de propósito, senso de autonomia e espiritualidade. Entre os componentes da felicidade mais citados por idosos estão, por um lado, as relações familiares, as relações sociais e o apoio social; por outro, a autorrealização, a autoaceitação, o estar bem consigo mesmo, a adaptação às condições atuais e a satisfação com a vida. Da mesma forma que a dicotomia objetivosubjetivo, os conceitos de hedonismo (busca de prazer e evitação do sofrimento) e eudaimonismo (busca de excelência pessoal) presidem as concepções de pessoas idosas sobre felicidade na velhice ${ }^{1-10}$. Elementos subjetivos e eudaimônicos prevalecem sobre os objetivos e hedônicos nas avaliações de idosos sobre a felicidade na velhice, concordando com pesquisas com objetivos similares ${ }^{1,11,12}$. Para as mulheres, felicidade é principalmente poder desfrutar do convívio com a família, manter relações de afeto e participar de atividades sociais ${ }^{13}$. Como relatado pelos estudos de revisão de Diener ${ }^{10,13}$, os homens mais do que as mulheres citam a manutenção de capacidades que lhes permitem a manutenção da independência e da autonomia e ter recursos para satisfazer as necessidades. A importância atribuída a elementos subjetivos e eudaimônicos tende a se manter e a atuar como recurso de proteção, em face do declínio associado ao processo de envelhecimento ${ }^{14}$.

Pesquisas transculturais discutiram a questão da universalidade dos significados de felicidade e concluiram que há diferenças transculturais quanto às prioridades de pessoas idosas em matéria de felicidade, mas não quanto à essência do conceito ou das aspirações ${ }^{11,12,15}$. Exemplificando, idosos entrevistados em Taiwan (China) indicaram como fontes de felicidade: gratificação da necessidade por respeito, harmonia nas relações interpessoais, realização no trabalho, levar a vida com tranquilidade, e derivar prazer da convivência com outros. Outros temas citados por eles são idênticos aos encontrados em amostras ocidentais: recursos materiais, autorrealização, prazer e afeto positivo, e saúde ${ }^{16}$. Estudo coreano identificou como fontes de felicidade a autorrealização, o pertencimento, a missão, o reconhecimento social, o desfrute, o sucesso material, e a parentalidade ${ }^{17}$. Idosos latinos vivendo nos E.U.A. valorizaram relações sociais
Keywords: Happiness.
Healthy Aging. Longevity. Quality of Life. 
harmoniosas, unidade da família, e fé/religiosidade, saúde física, autoestima, comunicação aberta com a família e os amigos, e segurança financeira ${ }^{18}$. $\mathrm{Na}$ Palestina, os idosos ressaltaram: senso de bem-estar, boa saúde física e mental, sentimentos positivos, independência, propósito de vida, contentamento e segurança financeira ${ }^{19}$. Exceção foi observada em amostra de idosos chineses que vivenciaram períodos de fome na infância e que apresentaram quantidade e qualidade de comida como as principais fontes de felicidade na velhice, sugerindo que certos tipos de experiências que testam os limites da dignidade humana podem provocar alterações nas prioridades vitais ${ }^{20}$.

Felicidade, qualidade de vida, saúde mental positiva, satisfação com a vida e bem-estar psicológico são termos correlatos, integrantes de um mesmo universo conceitual que pode ser definido em termos das condições que permitem uma vida ou uma velhice dignas de serem vividas. A decisão de utilizar um ou outro desses termos depende das tradições teóricas às quais se filiam as investigações ou as intervenções, e não de diferenças no significado dos termos ou nas metodologias usadas para investigá-los. Assim, na Psicologia predominam felicidade e bem-estar psicológico; nas Ciências Sociais, satisfação com a vida e bem-estar subjetivo, e na Epidemiologia, qualidade de vida, qualidade de vida em saúde e saúde mental positiva. Eventualmente, vários desses termos são usados ao mesmo tempo, caso do estudo canadense publicado em 2020, associado a um esforço de vigilância epidemiológica de saúde mental positiva, o qual utilizou cinco indicadores que se revelaram relacionados, mas independentes: autoavaliação da saúde mental, felicidade, satisfação com a vida, bemestar psicológico e bem-estar social ${ }^{21}$.

Boggatz $^{22}$ produziu uma análise conceitual do construto qualidade de vida na velhice, como veiculado por 208 artigos publicados entre 1992 e 2013. Dessa análise foram derivados três conceitos centrais: (a) satisfação das condições de vida (condições objetivas de vida de um idoso consideradas relevantes à uma boa vida e à uma velhice bem-sucedida, como por exemplo situação financeira, saúde e funcionalidade), (b) bem-estar subjetivo geral (ou bem-estar hedônico, indicado por satisfação com a vida) e (c) senso de completude com relação às principais dimensões da vida humana (de natureza eudaimônica), tais como busca de crescimento pessoal, autoconhecimento, autoaceitação, controle, autonomia, relações positivas com os outros e propósito. Van Leween et al. ${ }^{23}$ analisaram os dados de 48 estudos qualitativos sobre as opiniões de mais de 3.400 idosos de 11 países ocidentais e derivaram 11 categorias de qualidade de vida: autonomia, exercício de papeis e atividade, percepções de saúde, relações sociais, atitudes e adaptação, conforto emocional, espiritualidade, lar e vizinhança, e segurança financeira.

Tendo como objetivo construir uma escala de qualidade de vida para uso em grandes estudos longitudinais, Hyde et al. ${ }^{24}$ elaboraram uma medida escalar de qualidade de vida percebida, construto psicológico traduzido em quatro dimensões cognitivomotivacionais designadas pelo acrônimo CASP-19 (controle, autonomia, autorrealização e prazer) e operacionalizado por uma escala tipo Likert com 19 itens. As quatro dimensões foram teoricamente assumidas como necessidades ontológicas, isto é, inerentes ao Ser Humano. Conforme os autores, uma escala de qualidade de vida percebida não deve referir-se à saúde física, à funcionalidade e a condições materiais, porque estas variáveis são contextuais, não elementos constitutivos do construto. Originalmente produzida em inglês, a escala foi vertida para a língua portuguesa, submetida a validação semântico-cultural para uso em amostras de adultos e idosos brasileiros de 55 anos e mais, e submetida a estudos psicométricos que identificaram evidências de validade de construto e de validade convergente ${ }^{25}$.

Os aspectos subjetivos e eudaimônicos da experiência de envelhecimento têm potencial para atuar como recursos de proteção e como recursos motivacionais orientados ao autocuidado e à autoeducação, em face do aumento das perdas e das adversidades associadas à longevidade. Até onde nos é dado saber, são desconhecidos dados obtidos por pesquisas correlacionais, prospectivas ou longitudinais realizadas com amostras de idosos brasileiros, sobre relações entre medidas de qualidade de vida percebida e de significados de felicidade na velhice.

O objetivo deste estudo foi identificar associações entre significados atribuídos ao conceito "ser feliz na velhice" avaliado em linha 
de base (2008-2009), e qualidade de vida percebida avaliada no seguimento (2016-2017), em amostra de idosos recrutados na comunidade.

\section{MÉTODOS}

Os dados derivaram dos registros contidos nos bancos da linha de base (2008-2009) e do seguimento (2016-2017) do Estudo Fibra Campinas, um inquérito de base populacional com medidas repetidas, cujos objetivos foram levantar dados sobre fragilidade e investigar associações entre esta síndrome geriátrica com variáveis sociodemográficas, de saúde, funcionalidade e psicossociais. A amostra da linha de base $(\mathrm{N}=900)$ foi composta a partir de seleção aleatória de 90 dentre 900 setores censitários urbanos de Campinas, SP, Brasil, em cujos domicílios foram recrutados idosos de 65 anos e mais, em cotas de sexo e idade (65-69, 70-74, 75-79 e 80 anos e mais) representativas da população residente em cada um dos cinco distritos de saúde da cidade ${ }^{26}$. A amostra do seguimento foi composta pelos sobreviventes do estudo de linha de base que foram localizados e que responderam a medidas que incluíram a escala CASP-19 de qualidade de vida percebida ${ }^{27}$.

O fluxo das decisões adotadas para selecionar os participantes do estudo ora relatado foi o seguinte: (a) No banco da linha de base do Estudo Fibra Campinas (2008-2009; N=900 participantes com 65 anos e mais), exclusão de 211 indivíduos que apresentaram deficit cognitivo sugestivo de demência indicado por pontuação inferior à nota de corte no Mini-Exame do Estado Mental, ajustada pelos anos de escolaridade ${ }^{28,29}$, motivo pelo qual tornaram-se inelegíveis para responder ao item aberto sobre significados de felicidade na velhice. (b) Entre os 689 idosos que permaneceram na amostra da linha de base, exclusão de 18 que não tinham registro de resposta sobre felicidade na velhice. (c) Estudo do banco de dados do seguimento do Fibra Campinas (2016-2017) para saber quantos entre os 671 idosos com dados completos sobre o significado de felicidade na velhice na linha de base tinham registro das respostas à escala CASP19 de qualidade de vida percebida no seguimento. Por esse critério, foram selecionados 324 idosos. Foram identificadas 130 perdas por falecimento e
247 por não-localização. (d) Entre os 324 idosos sobreviventes localizados, exclusão de 83 por terem pontuação inferior à nota de corte no teste de rastreio cognitivo ${ }^{26,27}$. (e) Entre os 241 idosos que permaneceram na amostra, exclusão de 30 que não responderam a todos os itens da escala CASP-19. Assim, a amostra para este estudo ficou composta por 211 pessoas idosas com 74 anos e mais de idade.

As variáveis e medidas envolvidas nesta investigação foram as seguintes: (a) Significados atribuídos ao conceito "ser feliz na velhice", na presença de um item aberto que perguntava a esse respeito, na linha de base ${ }^{27}$. (b) Qualidade de vida percebida indicada pela escala CASP-19 aplicada no seguimento $^{28}$. (c) Sexo e idade: os idosos podiam responder sexo masculino ou sexo feminino e deviam nomear o dia, o mês e o ano do seu nascimento. Foi considerada como idade de referência, aquela informada no seguimento.

A variável qualidade de vida percebida foi avaliada por meio da CASP-19, acrônimo de controle, autonomia, autorrealização e prazer, que designa uma escala de 19 itens que expressam necessidades psicológicas básicas não hierarquizadas. Essas são consideradas ontológicas por Hyde et al. ${ }^{24}$, orientadoras da personalidade e do desenvolvimento. Os itens são tipo Likert, ancorados por quatro intensidades $(0=$ nada, $1=$ um pouco, $2=$ muito, $e$ $3=$ muitíssimo). A pontuação total varia de 0 a $57^{24,25}$.

Análises fatoriais exploratórias e confirmatórias do conteúdo da escala CASP-19 traduzida e culturalmente adaptada para o português revelaram uma estrutura de significados latentes expressos em dois fatores, a qual foi considerada neste estudo: fator 1 - Autorrealização e Prazer e fator 2 - Controle e Autonomia ${ }^{25}$.

Os testes de Mann Whitney, Kruskal-Wallis e Dunn foram utilizados para comparar as distribuições de frequência das pontuações na escala CASP-19 total e em seus dois fatores, uma vez que os testes prévios de normalidade demonstraram que os dados eram não-paramétricos. Como a CASP-19 não tem notas de corte decididas por critérios estatísticos, nem seus resultados são confrontáveis com padrões-ouro, o estabelecimento dos níveis de desempenho na escala é normalmente feito com base nas pontuações obtidas 
pelas próprias amostras. Neste estudo, utilizamos como critério o valor da mediana das distribuições das pontuações na escala total e nos itens dos dois fatores. Foram comparadas as frequências dos participantes com pontuações superiores e inferiores à mediana de cada distribuição considerando-se os significados de felicidade na velhice, sexo e idade.

Os significados de "ser feliz na velhice" foram obtidos em situação de entrevista, solicitando-se aos participantes que falassem livremente sobre o assunto. As respostas foram registradas literalmente no formulário da pesquisa e depois transcritas para o banco de dados ${ }^{26}$. O corpus formado pelos registros textuais das respostas orais dos idosos foi submetido à análise de conteúdo e à contagem das categorias, para comparação com os dados da CASP-19.

A análise de conteúdo é uma técnica categorial e inferencial de análise da comunicação, que se vale de procedimentos sistemáticos e objetivos de descrição, classificação, quantificação e qualificação das mensagens. Foi considerada como unidade de análise qualquer emissão completa e inteligível, com qualquer extensão ou nível de complexidade linguística, em resposta à questão aberta. Categoria foi definida como uma classe de significados que compartilham pelo menos um elemento comum, que os diferencia de outra classe; os temas, como conceitos supra ordenados que aglutinam um conjunto de categorias ${ }^{30,31}$.

A análise foi realizada por três pesquisadores informados sobre o método e sobre os objetivos da análise, e que conheciam os resultados de pesquisas similares $^{4,5}$. Trabalhando independentemente e depois em conjunto, ocasião em que se submeteram a uma exigência de acordo inter-examinadores da ordem de $100 \%$, os analistas derivaram quatro temas e 11 categorias de significado, apoiados nos conceitos de bem-estar hedônico e eudaimônico ${ }^{1}$ e no modelo teórico de bem-estar psicológico de Ryff ${ }^{14,32}$. Os dados foram traduzidos em registros de ocorrência e não ocorrência das categorias abstraídas da resposta de cada idoso, e foram submetidas a contagem. Os testes Qui-quadrado de Pearson e Exato de Fisher foram usados para comparar frequências das variáveis categóricas produzidas pela análise de conteúdo.
Análises de regressão logística univariada e múltipla, que adotou o método step wise de seleção de variáveis foram usadas para verificar as associações entre as variáveis de interesse. Os resultados dos testes estatísticos foram considerados significantes para valor de $p<0,05$. As análises estatísticas foram realizadas por meio dos pacotes SAS (Statistical Analysis System), versão 9.4., e SPSS, versão 21.0.

Os participantes assinaram termos de consentimento livre esclarecido relativo aos objetivos, procedimentos e compromissos éticos da equipe, em ambos os tempos de medida. Os projetos foram aprovados pelo Comitê de Ética da Universidade Estadual de Campinas pelos pareceres № 208/2007, de 22/5/2007 e No 1.332.651, de 23/11/2015. O projeto do presente estudo foi aprovado em 05/11/2019, pelo parecer No 3.684.200.

\section{RESULTADOS}

A amostra foi composta por 60 homens e 151 mulheres com idade média de $81,0 \pm 4,3$ anos no seguimento; o grupo de idade mais numeroso foi o de 80 a 84 anos. A análise de conteúdo sobre o conceito "ser feliz na velhice" identificou quatro temas (saúde e funcionalidade, bem-estar psicológico, relações interpessoais e recursos materiais) e 11 categorias a eles subordinadas (Tabela 1).

A Tabela 2 apresenta os dados das variáveis sociodemográficas, as frequências de respostas nas categorias de significado de felicidade na velhice e os valores das medianas, das médias e das distâncias interquartílicas na escala CASP-19 e nos fatores Autorrealização/Prazer e Controle/Autonomia. As categorias de significados do conceito "ser feliz na velhice" com maior frequência de ocorrências foram saúde física, relações familiares, autodesenvolvimento e satisfação e prazer. Cognição e religiosidade/ espiritualidade, os de menor frequência. No tema bem-estar psicológico destacaram-se as três categorias relativas ao bem-estar eudaimônico $(68,0 \%$ das menções), que superaram em frequência as relativas a bem-estar hedônico (satisfação e prazer). Foram altos os valores das médias e das medianas na escala CASP-19 e nos dois fatores. 
No grupo que pontuou acima da mediana na CASP-19 foi observada uma frequência significativamente mais alta de idosos que não associaram "ser feliz na velhice" nem com saúde e funcionalidade e nem com recursos materiais do que de idosos que fizeram essas associações. Entre estes, predominaram idosos com pontuação inferior à mediana na escala de qualidade de vida percebida. Foram observados mais idosos que pontuaram alto na CASP-19 entre os que mencionaram bem-estar psicológico, satisfação e prazer, relações interpessoais, relações familiares e relações sociais do que de idosos que não mencionaram esses significados. Nesse grupo predominaram participantes com pontuação inferior à mediana na CASP-19. Essas relações se repetiram para as relações entre o fator autorrealização e prazer da escala de qualidade de vida percebida e os significados saúde e funcionalidade, recursos materiais, satisfação e prazer, relações sociais e suporte social. Houve mais idosos de 80 a 84 anos do que de 85 anos e mais com pontuação superior à mediana no fator autorrealização e prazer. Entre estes últimos preponderaram pontuações mais baixas no fator (Tabela 3).

Tabela 1. Temas e categorias latentes aos significados atribuídos pelos idosos ao conceito "ser feliz na velhice" ( $\mathrm{n}=211)$. Campinas, SP, 2020.

Tema 1. Saúde e funcionalidade

\section{Categorias:}

1.1. Saúde física. Status de saúde traduzido em diagnósticos médicos, sinais e sintomas; estilos de vida saudáveis. Ex: Ter cuidados consigo mesmo.

1.2. Atividade. Envolvimento vital, participação social, produtividade, energia. Ex: Ter vontade e força para trabalhar.

1.3. Independência e autonomia. Capacidade física e mental que permite controle sobre o ambiente; autogoverno, autodeterminação. Ex: Fazer o que quer, sem receber ajuda.

1.4. Cognição. Funções cognitivas básicas preservadas, permitindo solução de problemas, discernimento, raciocínio julgamento e autonomia. Ex: Não queria perder minha memória.

Tema 2. Bem-estar psicológico

\section{Categorias:}

2.1. Satisfação e prazer. Produtos do senso de que se tem vida boa e feliz, em concordância com os valores e as expectativas pessoais e sociais. Ex: Ter sossego, ter paz e alegria, família.

2.2. Religiosidade e espiritualidade. O sagrado e o transcendente como fontes de significado existencial e de senso de pertencimento e como recursos de enfrentamento. Ex: Primeiro e em tudo servir a Deus.

2.3. Enfrentamento com base na emoção. Manejo de situações estressantes por meio de estratégias cognitivoemocionais que protegem a autoestima e poupam recursos pessoais. Ex: Estar bem consigo mesmo.

2.4. Autodesenvolvimento. Investimentos em autoconhecimento e auto aceitação busca de excelência pessoal, senso de propósito e senso de autorrealização. Ex: Aprender a lidar com as dificuldades.

Tema 3. Relações interpessoais

Categorias:

3.1. Relações familiares. O núcleo familiar como fonte de reconhecimento, pertencimento, valorização, respeito, proteção, afeto, segurança, apoio e satisfação. Ex: Família unida.

3.2. Relações sociais. O grupo como fonte de reconhecimento, pertencimento, valorização, proteção, afeto, segurança e satisfação. Ex: É viver bem ... amar, viver bem com os amigos.

3.3. Suporte social. Relações de dar e receber afeto, bens materiais, informação, ajuda instrumental e auxílio na solução de problemas. Ex: É ter uma pessoa que cuide da gente, poder cuidar.

Tema 4. Recursos materiais

Posse de dinheiro, objetos e oportunidades que facilitam o alcance do bem-estar, de acordo com os valores individuais e sociais. Ex: Dinheiro para ir ao médico, para comer, comprar remédio. 
Tabela 2. Sumário descritivo da amostra (n=211). Campinas, SP, 2020.

\begin{tabular}{|c|c|c|c|c|}
\hline & $\mathrm{n}(\%)$ & $\begin{array}{l}\text { Médias } \\
\text { (desvios-padrão) }\end{array}$ & Medianas & $\begin{array}{l}\text { Distâncias } \\
\text { interquartílicas }\end{array}$ \\
\hline \multicolumn{5}{|l|}{ Sexo } \\
\hline Masculino & $60(33,2)$ & & & \\
\hline Feminino & $151(66,8)$ & & & \\
\hline Idade (anos) & & $81,0 \pm 4,3$ & 81,0 & 6,0 \\
\hline $70-79$ & $71(33,7)$ & & & \\
\hline $80-84$ & $97(46,4)$ & & & \\
\hline$\geq 85$ & $42(19,9)$ & & & \\
\hline
\end{tabular}

Ocorrências de categorias de significado

de "ser feliz na velhice"

T1. Saúde e funcionalidade

C1.1. Saúde física

$122(56,2)$

C1.2 Atividade

$40(18,4)$

C1.3. Independência /autonomia

$48(22,2)$

C1.4. Cognição

$7(3,2)$

T2. Bem-estar psicológico

C2.1. Satisfação e prazer

$66(32,0)$

C2.2. Religiosidade/espiritualidade

$29(14,1)$

C2.3. Enfrentamento base emoção

$42(10,4)$

C2.4. Autodesenvolvimento

$69(33,5)$

T3. Relações interpessoais

C3.1. Relações familiares

$80(41,5)$

C3.2. Relações sociais

$54(28,0)$

C3.3. Suporte social

T4. Recursos materiais $56(26,5)$

Idosos conforme pontuação em qualidade de vida percebida

Escala CASP-19 total

$40,2 \pm 8,7 \quad 40,0 \quad 10,0$

Com pontuação abaixo da mediana

$101(47,8)$

Com pontuação acima da mediana $110(52,2)$

Fator 1: Autorrealização / Prazer

$26,6 \pm 7,3 \quad 26,0 \quad 10,0$

Com pontuação abaixo da mediana

$98(46,4)$

Com pontuação acima da mediana $113(53,6)$

Fator 2: Controle / Autonomia

Com pontuação abaixo da mediana

$$
4,4 \pm 3,4
$$

4,0

4,0

Com pontuação acima da mediana

$101(47,8)$

$\mathrm{T}=$ Tema; $\mathrm{C}=$ Categoria.

$110(52,2)$ 
Tabela 3. Associações entre pontuação superior à mediana na escala CASP-19 de qualidade de vida percebida, considerando os significados de "ser feliz na velhice", sexo e idade dos participantes (n=211). Campinas, SP, 2020.

\begin{tabular}{|c|c|c|c|c|c|c|c|}
\hline \multirow{2}{*}{ Variáveis } & \multirow{2}{*}{$\mathrm{n}(\%)$} & \multicolumn{2}{|c|}{ Escala CASP-19 } & \multicolumn{2}{|c|}{$\begin{array}{l}\text { Fator } 1 . \\
\text { Autorrealização/Prazer }\end{array}$} & \multicolumn{2}{|c|}{$\begin{array}{l}\text { Fator } 2 . \\
\text { Controle/Autonomia }\end{array}$} \\
\hline & & $\begin{array}{l}\leq 39,0 \\
(n=101)\end{array}$ & $\begin{array}{l}\geq 40,0 \\
(n=110)\end{array}$ & $\begin{array}{l}\leq 25,0 \\
(\mathrm{n}=98)\end{array}$ & $\begin{array}{l}\geq 26,0 \\
(n=113)\end{array}$ & $\begin{array}{l}\leq 3,0 \\
(n=101)\end{array}$ & $\begin{array}{l}\geq 4,0 \\
(n=110)\end{array}$ \\
\hline \multicolumn{8}{|l|}{$\begin{array}{l}\text { Significados de "ser feliz } \\
\text { na velhice" }\end{array}$} \\
\hline \multicolumn{2}{|l|}{ T1. Saúde e funcionalidade } & \multicolumn{2}{|c|}{$p=\mathbf{0 , 0 3 3}$} & \multicolumn{2}{|c|}{$p=0,038$} & \multicolumn{2}{|c|}{$p=0,383$} \\
\hline Não & $69(32,7)$ & 33,3 & 66,7 & 36,2 & 63,8 & 52,2 & 47,8 \\
\hline $\operatorname{Sim}$ & $142(67,3)$ & 54,9 & 45,1 & 51,4 & 48,6 & 45,8 & 54,2 \\
\hline \multicolumn{2}{|l|}{ C1.1. Saúde física } & \multicolumn{2}{|c|}{$p=0,065$} & \multicolumn{2}{|c|}{$p=0,226$} & \multicolumn{2}{|c|}{$p=0,132$} \\
\hline Não & $89(42,2)$ & 40,0 & 59,6 & 41,6 & 58,4 & 53,9 & 46,1 \\
\hline $\operatorname{Sim}$ & $122(57,8)$ & 53,3 & 46,7 & 50,0 & 50,0 & 43,4 & 56,6 \\
\hline \multicolumn{2}{|l|}{ C1.2. Atividade } & \multicolumn{2}{|c|}{$p=0,515$} & \multicolumn{2}{|c|}{$p=0,364$} & \multicolumn{2}{|c|}{$p=0,450$} \\
\hline Não & $171(81,0)$ & 46,8 & 53,2 & 47,9 & 52,1 & 49,1 & 50,9 \\
\hline $\operatorname{Sim}$ & $40(19,0)$ & 52,5 & 47,5 & 40,0 & 60,0 & 42,5 & 57,5 \\
\hline \multicolumn{2}{|l|}{$\begin{array}{l}\text { C1.3. Independência/ } \\
\text { autonomia }\end{array}$} & \multicolumn{2}{|c|}{$p=0,099$} & \multicolumn{2}{|c|}{$p=0,923$} & \multicolumn{2}{|c|}{$p=0,748$} \\
\hline Não & $163(77,2)$ & 44,8 & 55,2 & 46,6 & 53,4 & 48,5 & 51,5 \\
\hline $\operatorname{Sim}$ & $48(22,7)$ & 58,3 & 41,7 & 45,8 & 54,2 & 45,8 & 54,2 \\
\hline \multicolumn{2}{|l|}{ C1.4. Cognição } & \multicolumn{2}{|c|}{$p=0,056$} & \multicolumn{2}{|c|}{$p=0,051$} & \multicolumn{2}{|c|}{$p=1,000$} \\
\hline Não & $204(96,7)$ & 46,6 & 53,4 & 45,1 & 54,9 & 48,0 & 52,0 \\
\hline Sim & $7(3,3)$ & 85,7 & 14,3 & 85,7 & 14,3 & 42,9 & 57,1 \\
\hline \multicolumn{2}{|l|}{ T2. Bem-estar psicológico } & &, 006 & &, 127 & & 598 \\
\hline Não & $113(53,5)$ & 56,6 & 43,4 & 51,3 & 48,7 & 49,6 & 50,4 \\
\hline $\operatorname{Sim}$ & $98(46,5)$ & 37,8 & 62,2 & 40,8 & 59,2 & 45,9 & 54,1 \\
\hline C2.1. Satisfação e prazer & & &, 004 & &, 048 & & 172 \\
\hline Não & $145(68,7)$ & 54,5 & 45,5 & 51,0 & 49,0 & 51,0 & 49,0 \\
\hline Sim & $66(31,3)$ & 33,3 & 66,7 & 36,4 & 63,6 & 40,9 & 59,1 \\
\hline $\begin{array}{l}\text { C2.2. Religiosidade/ } \\
\text { espiritualidade }\end{array}$ & & & 0,724 & &, 832 & & 724 \\
\hline Não & $182(86,3)$ & 48,3 & 51,7 & 46,1 & 53,8 & 48,3 & 51,7 \\
\hline Sim & $29(13,7)$ & 44,8 & 55,2 & 48,3 & 51,7 & 44,8 & 55,2 \\
\hline $\begin{array}{l}\text { C2.3. Enfrentamento base } \\
\text { Emoção }\end{array}$ & & & 0,703 & &, 861 & & 757 \\
\hline Não & $169(80,0)$ & 48,5 & 51,5 & 46,7 & 53,2 & 47,3 & 52,7 \\
\hline Sim & $42(20,0)$ & 45,2 & 54,8 & 45,2 & 54,7 & 50,0 & 50,0 \\
\hline C2.4. Autodesenvolvimento & & & 0,077 & &, 138 & & 993 \\
\hline Não & $142(67,3)$ & 52,1 & 47,9 & 50,0 & 50,0 & 47,9 & 52,1 \\
\hline $\operatorname{Sim}$ & $69(32,7)$ & 39,1 & 60,9 & 39,1 & 60,9 & 47,8 & 52,2 \\
\hline T3. Relações interpessoais & & & 0,011 & &, 120 & & 820 \\
\hline Não & $102(48,3)$ & 56,9 & 43,1 & 52,0 & 48,0 & 47,1 & 52,9 \\
\hline $\operatorname{Sim}$ & $109(51,7)$ & 39,4 & 60,6 & 41,3 & 58,7 & 48,6 & 51,4 \\
\hline
\end{tabular}


Continuação da Tabela 3

\begin{tabular}{|c|c|c|c|c|c|c|c|}
\hline \multirow{2}{*}{ Variáveis } & \multirow{2}{*}{$\mathrm{n}(\%)$} & \multicolumn{2}{|c|}{ Escala CASP-19 } & \multicolumn{2}{|c|}{$\begin{array}{l}\text { Fator } 1 . \\
\text { Autorrealização/Prazer }\end{array}$} & \multicolumn{2}{|c|}{$\begin{array}{l}\text { Fator } 2 . \\
\text { Controle/Autonomia }\end{array}$} \\
\hline & & $\begin{array}{l}\leq 39,0 \\
(n=101)\end{array}$ & $\begin{array}{l}\geq 40,0 \\
(n=110)\end{array}$ & $\begin{array}{l}\leq 25,0 \\
(\mathrm{n}=98)\end{array}$ & $\begin{array}{l}\geq 26,0 \\
(n=113)\end{array}$ & $\begin{array}{l}\leq 3,0 \\
(n=101)\end{array}$ & $\begin{array}{l}\geq 4,0 \\
(n=110)\end{array}$ \\
\hline \multicolumn{8}{|l|}{$\begin{array}{l}\text { Significados de "ser feliz } \\
\text { na velhice" }\end{array}$} \\
\hline \multicolumn{2}{|l|}{ C3.1. Relações familiares } & \multicolumn{2}{|c|}{$p=0,038$} & \multicolumn{2}{|c|}{$p=0,142$} & \multicolumn{2}{|c|}{$p=0,628$} \\
\hline Não & $131(62,1)$ & 53,4 & 46,6 & 50,4 & 49,6 & 46,6 & 53,4 \\
\hline $\operatorname{Sim}$ & $80(37,9)$ & 38,7 & 61,3 & 40,0 & 60,0 & 50,0 & 50,0 \\
\hline \multicolumn{2}{|l|}{ C3.2. Relações sociais } & \multicolumn{2}{|c|}{$p=0,031$} & \multicolumn{2}{|c|}{$p=0,025$} & \multicolumn{2}{|c|}{$p=0,190$} \\
\hline Não & $157(74,4)$ & 52,2 & 47,8 & 51 & 49,0 & 45,2 & 54,8 \\
\hline $\operatorname{Sim}$ & $54(25,6)$ & 35,2 & 64,8 & 33,3 & 66,7 & 55,6 & 44,4 \\
\hline \multicolumn{2}{|l|}{ C3.3. Suporte social } & \multicolumn{2}{|c|}{$p=0,108$} & \multicolumn{2}{|c|}{$p=\mathbf{0 , 0 2 3}$} & \multicolumn{2}{|c|}{$p=0,703$} \\
\hline Não & $152(72,0)$ & 51,3 & 48,7 & 51,3 & 48,7 & 48,7 & 51,3 \\
\hline $\operatorname{Sim}$ & $59(28,0)$ & 39,0 & 61,0 & 33,9 & 66,1 & 45,8 & 54,2 \\
\hline \multicolumn{2}{|l|}{ T4. Recursos materiais } & \multicolumn{2}{|c|}{$p=0,025$} & \multicolumn{2}{|c|}{$p=0,212$} & \multicolumn{2}{|c|}{$p=0,709$} \\
\hline Não & $155(73,5)$ & 43,2 & 56,8 & 43,9 & 56,1 & 47,1 & 52,9 \\
\hline Sim & $56(26,5)$ & 60,7 & 39,3 & 53,6 & 46,4 & 50,0 & 50,0 \\
\hline \multicolumn{2}{|l|}{ Sexo } & \multicolumn{2}{|c|}{$p=0,486$} & \multicolumn{2}{|c|}{$p=0,206$} & \multicolumn{2}{|c|}{$p=0,599$} \\
\hline Masculino & $60(28,4)$ & 51,7 & 48,3 & 53,3 & 46,7 & 45,0 & 55,0 \\
\hline Feminino & $151(71,5)$ & 46,4 & 53,6 & 43,7 & 56,3 & 49,0 & 51,0 \\
\hline \multicolumn{2}{|l|}{ Idade (anos) } & \multicolumn{2}{|c|}{$p=0,228$} & \multicolumn{2}{|c|}{$p=\mathbf{0 , 0 3 3}$} & \multicolumn{2}{|c|}{$p=0,377$} \\
\hline $70-79$ & $71(33,8)$ & 53,5 & 46,5 & 50,7 & 49,3 & 43,7 & 56,3 \\
\hline $80-84$ & $97(46,2)$ & 41,2 & 58,8 & 37,1 & 62,9 & 47,4 & 52,6 \\
\hline$\geq 85$ & $42(20,0)$ & 52,4 & 47,6 & 59,5 & 40,5 & 57,1 & 42,9 \\
\hline
\end{tabular}

$\mathrm{T}=$ Tema $\mathrm{C}=$ Categoria; Diferença estatisticamente significativa entre os grupos se $p$-valor $<0,05$, para o teste Qui-quadrado de Pearson.

Na análise de regressão logística simples, os idosos que mencionaram os temas saúde e funcionalidade e recursos materiais apresentaram-se com menores chances de pontuar acima da mediana na CASP19. Os que mencionaram bem-estar psicológico, satisfação e prazer, relações interpessoais e relações familiares apresentaram-se com maiores chances de pontuar acima da mediana na escala. Conforme dados da análise de regressão logística múltipla, idosos que não mencionaram recursos materiais ou saúde e funcionalidade tiveram maior probabilidade de pontuar acima da mediana na CASP-19 do que aqueles que mencionaram esses significados. Idosos que mencionaram relações interpessoais tiveram maior probabilidade de pontuar acima da mediana na CASP-19, do que os que não mencionaram (Tabela 4).
A análise de regressão logística univariada para as associações entre pontuação superior à mediana no fator autorrealização/prazer e significado de "ser feliz na velhice" revelou resultados similares aos obtidos para a escala total. Em comparação com as pessoas idosas que não mencionaram, as que mencionaram relações sociais, saúde e funcionalidade, e satisfação e prazer tiveram maior chance de pontuar acima da mediana nos itens do fator autorrealização/prazer. A análise de regressão logística múltipla mostrou associações significativas entre pontuação superior à mediana no fator autorrealização e prazer e presença de menções a suporte social, ausência de menções à saúde e funcionalidade e idade de 80 a 84 anos (Tabela 5). Foram realizadas análises de regressão para o fator 2 da CASP-19 (controle/autonomia), sem resultados estatisticamente significativos. 
Tabela 4. Análises de regressão logística das pontuações superiores à mediana na escala CASP-19, dos significados de "ser feliz na velhice", sexo e idade dos participantes (n=211). Campinas, SP, 2020.

\begin{tabular}{|c|c|c|c|c|c|c|}
\hline \multirow{2}{*}{$\begin{array}{l}\text { Variáveis } \\
\text { Significados de "Ser feliz na velhice" }\end{array}$} & \multicolumn{3}{|c|}{$\begin{array}{l}\text { Análise de regressão logística } \\
\text { univariada }\end{array}$} & \multicolumn{3}{|c|}{$\begin{array}{l}\text { Análise de regressão logística } \\
\text { múltipla }\end{array}$} \\
\hline & ${ }^{*} \mathrm{OR}$ & ${ }^{* *} \mathrm{IC} 95 \%$ OR & $p$-valor & ${ }^{*} \mathrm{OR}$ & ${ }^{* *} \mathrm{IC} 95 \%$ OR & $p$-valor \\
\hline \multicolumn{7}{|l|}{ T1. Saúde e Funcionalidade } \\
\hline Não & 1,00 & --- & --- & 1,00 & --- & --- \\
\hline Sim & 0,41 & $0,23-0,75$ & 0,004 & 0,44 & $0,24-0,82$ & 0,010 \\
\hline \multicolumn{7}{|l|}{ T2. Bem-Estar Psicológico } \\
\hline Não & 1,00 & --- & --- & & & \\
\hline Sim & 2,15 & $1,24-3,74$ & 0,007 & & & \\
\hline \multicolumn{7}{|l|}{ T3. Relações Interpessoais } \\
\hline Não & 1,00 & --- & --- & 1,00 & --- & --- \\
\hline $\operatorname{Sim}$ & 2,02 & $1,17-3,50$ & 0,012 & 1,92 & $1,08-3,41$ & 0,027 \\
\hline \multicolumn{7}{|l|}{ T4. Recursos Materiais } \\
\hline Não & 1,00 & --- & --- & 1,00 & --- & --- \\
\hline $\operatorname{Sim}$ & 0,49 & $0,26-0,92$ & 0,026 & 0,43 & $0,23-0,83$ & 0,012 \\
\hline \multicolumn{7}{|l|}{ C1.1 Saúde física } \\
\hline Não & 1,00 & --- & --- & & & \\
\hline $\operatorname{Sim}$ & 0,60 & $0,34-1,04$ & 0,066 & & & \\
\hline \multicolumn{7}{|l|}{ C1.2 Atividade } \\
\hline Não & 1,00 & --- & --- & & & \\
\hline Sim & 0,80 & $0,40-1,59$ & 0,515 & & & \\
\hline \multicolumn{7}{|l|}{ C1.3 Independência/autonomia } \\
\hline Não & 1,00 & --- & --- & & & \\
\hline Sim & 0,58 & $0,30-1,11$ & 0,101 & & & \\
\hline \multicolumn{7}{|l|}{ C1.4 Cognição } \\
\hline Não & 1,00 & --- & --- & & & \\
\hline $\operatorname{sim}$ & 0,15 & $0,02-1,23$ & 0,077 & & & \\
\hline \multicolumn{7}{|l|}{ C2.1 Satisfação e prazer } \\
\hline Não & 1,00 & --- & --- & & & \\
\hline Sim & 2,39 & $1,30-4,39$ & 0,005 & & & \\
\hline \multicolumn{7}{|l|}{ C2.2 Religiosidade/espiritualidade } \\
\hline Não & 1,00 & --- & --- & & & \\
\hline Sim & 1,15 & $0,52-2,53$ & 0,724 & & & \\
\hline \multicolumn{7}{|l|}{ C.2.3 Enfrentamento/emoção } \\
\hline Não & 1,00 & --- & --- & & & \\
\hline Sim & 1,14 & $0,58-2,25$ & 0,704 & & & \\
\hline \multicolumn{7}{|l|}{ C.2.4 Autodesenvolvimento } \\
\hline Não & 1,00 & --- & --- & & & \\
\hline Sim & 1,69 & $0,94-3,04$ & 0,078 & & & \\
\hline \multicolumn{7}{|l|}{ C3.1 Relações familiares } \\
\hline Não & 1,00 & --- & --- & & & \\
\hline Sim & 1,81 & 1,03-3,19 & 0,039 & & & \\
\hline \multicolumn{7}{|l|}{ C3.2 Relações sociais } \\
\hline Não & 1,00 & --- & --- & & & \\
\hline Sim & 2,01 & $1,06-3,82$ & 0,032 & & & \\
\hline
\end{tabular}


Continuação da Tabela 4

\begin{tabular}{llll}
\hline Variáveis & $\begin{array}{l}\text { Análise de regressão logística } \\
\text { univariada }\end{array}$ & $\begin{array}{l}\text { Análise de regressão logística } \\
\text { múltipla }\end{array}$ \\
\hline $\begin{array}{l}\text { Sexo } \\
\text { Masculino }\end{array}$ & 1,00 & -- & --- \\
Feminino & 1,24 & $0,68-2,25$ & 0,486 \\
Idade (anos) & & & \\
$70-79$ & 1,00 & --- & -- \\
$80-84$ & 1,64 & $0,89-3,04$ & 0,116 \\
$\geq 85$ & 1,05 & $0,49-2,25$ & 0,907 \\
\hline
\end{tabular}

$\mathrm{T}=$ Tema; $\mathrm{C}=$ Categoria. $\mathrm{OR}^{*}=$ razões de chance para alta pontuação: 110 com pontuação superior à mediana e 101 com pontuação inferior à mediana. IC $95 \%$ OR** = Intervalo de confiança de $95 \%$ para a razão de chance; $\mathrm{p}$-valor do teste de Wald estatisticamente significativo se $\mathrm{p}<0,05$.

Tabela 5. Análises de regressão logística das pontuações superiores à mediana no Fator 1 (Autorrealização/ Prazer) da versão em português da escala CASP-19, dos significados de "ser feliz na velhice", sexo e idade dos participantes (n=211). Campinas, SP, 2020.

\begin{tabular}{|c|c|c|c|c|c|c|}
\hline \multirow[t]{2}{*}{ Variáveis } & \multicolumn{3}{|c|}{$\begin{array}{l}\text { Análise de regressão logística } \\
\text { univariada }\end{array}$} & \multicolumn{3}{|c|}{$\begin{array}{l}\text { Análise de regressão logística } \\
\text { múltipla }\end{array}$} \\
\hline & ${ }^{*} \mathrm{OR}$ & ${ }^{* *} \mathrm{IC} 95 \%$ OR & $p$-valor & ${ }^{*} \mathrm{OR}$ & ${ }^{* *} \mathrm{IC} 95 \%$ OR & $p$-valor \\
\hline \multicolumn{7}{|c|}{ Significados de "ser feliz na velhice" } \\
\hline \multicolumn{7}{|c|}{ T1. Saúde e Funcionalidade } \\
\hline Não & 1,00 & --- & --- & 1,00 & --- & --- \\
\hline Sim & 0,54 & $0,30-0,97$ & 0,039 & 0,51 & $0,27-0,95$ & 0,035 \\
\hline \multicolumn{7}{|c|}{ T2. Bem-Estar Psicológico } \\
\hline Não & 1,00 & --- & --- & & & \\
\hline Sim & 1,53 & $0,89-2,64$ & 0,128 & & & \\
\hline \multicolumn{7}{|c|}{ T3 Relações Interpessoais } \\
\hline Não & 1,00 & --- & --- & & & \\
\hline Sim & 1,54 & $0,89-2,65$ & 0,121 & & & \\
\hline \multicolumn{7}{|c|}{ T4. Recursos Materiais } \\
\hline Não & 1,00 & --- & --- & & & \\
\hline $\operatorname{Sim}$ & 0,68 & $0,37-1,25$ & 0,213 & & & \\
\hline \multicolumn{7}{|c|}{ C1.1. Saúde Física } \\
\hline Não & 1,00 & --- & --- & & & \\
\hline $\operatorname{Sim}$ & 0,71 & $0,41-1,24$ & 0,226 & & & \\
\hline \multicolumn{7}{|c|}{ C1.2. Atividade } \\
\hline Não & 1,00 & --- & ---- & & & \\
\hline Sim & 1,38 & $0,69-2,78$ & 0,365 & & & \\
\hline \multicolumn{7}{|c|}{ C1.3. Independência/Autonomia } \\
\hline Não & 1,00 & --- & --- & & & \\
\hline Sim & 1,03 & $0,54-1,97$ & 0,923 & & & \\
\hline \multicolumn{7}{|c|}{ C1.4. Cognição } \\
\hline Não & 1,00 & --- & --- & & & \\
\hline Sim & 0,14 & $0,02-1,16$ & 0,068 & & & \\
\hline \multicolumn{7}{|c|}{ C2.1. Satisfação e Prazer } \\
\hline Não & 1,00 & --- & --- & & & \\
\hline Sim & 1,82 & $1,01-3,32$ & 0,049 & & & \\
\hline
\end{tabular}


Continuação da Tabela 5

\begin{tabular}{|c|c|c|c|c|c|c|}
\hline \multirow[t]{2}{*}{ Variáveis } & \multicolumn{3}{|c|}{$\begin{array}{l}\text { Análise de regressão logística } \\
\text { univariada }\end{array}$} & \multicolumn{3}{|c|}{$\begin{array}{l}\text { Análise de regressão logística } \\
\text { múltipla }\end{array}$} \\
\hline & ${ }^{*} \mathrm{OR}$ & ${ }^{* *} \mathrm{IC} 95 \%$ OR & $p$-valor & ${ }^{*} \mathrm{OR}$ & ${ }^{* *} \mathrm{IC} 95 \%$ OR & $p$-valor \\
\hline \multicolumn{7}{|c|}{ Significados de "ser feliz na velhice" } \\
\hline \multicolumn{7}{|c|}{ C2.2. Religiosidade/Espiritualidade } \\
\hline Não & 1,00 & --- & --- & & & \\
\hline $\operatorname{Sim}$ & 0,92 & $0,42-2,01$ & 0,831 & & & \\
\hline \multicolumn{7}{|c|}{ C2.3. Enfrentamento/emoção } \\
\hline Não & 1,00 & --- & --- & & & \\
\hline $\operatorname{Sim}$ & 1,06 & $0,54-2,09$ & 0,861 & & & \\
\hline \multicolumn{7}{|c|}{ C2.4. Autodesenvolvimento } \\
\hline Não & 1,00 & --- & --- & & & \\
\hline $\operatorname{Sim}$ & 1,56 & $0,87-2,79$ & 0,139 & & & \\
\hline \multicolumn{7}{|c|}{ C3.1. Relações Familiares } \\
\hline Não & 1,00 & --- & --- & & & \\
\hline Sim & 1,52 & $0,87-2,68$ & 0,143 & & & \\
\hline \multicolumn{7}{|c|}{ C3.2. Relações Sociais } \\
\hline Não & 1,00 & --- & --- & & & \\
\hline $\operatorname{Sim}$ & 2,08 & $1,09-3,97$ & 0,020 & & & \\
\hline \multicolumn{7}{|c|}{ C3.3. Suporte Social } \\
\hline Não & 1,00 & --- & --- & 1,00 & --- & --- \\
\hline Sim & 2,06 & $1,10-3,84$ & 0,024 & 2,19 & $1,00-2,19$ & 0,019 \\
\hline \multicolumn{7}{|l|}{ Sexo } \\
\hline Masculino & 1,00 & --- & --- & & & \\
\hline Feminino & 1,47 & $0,81-2,68$ & 0,207 & & & \\
\hline \multicolumn{7}{|c|}{ Idade (anos) } \\
\hline $74-79$ & 1,00 & --- & --- & 1,00 & --- & --- \\
\hline $80-84$ & 1,74 & $0,94-3,24$ & 0,080 & 1,93 & $1,01-3,68$ & 0,047 \\
\hline$\geq 85$ & 0,70 & $0,32-1,51$ & 0,364 & 0,58 & $0,26-1,32$ & 0,194 \\
\hline
\end{tabular}

$\mathrm{T}=$ Tema; $\mathrm{C}=$ Categoria; ${ }^{*} \mathrm{OR}=$ razões de chance para alta pontuação: 110 com pontuação superior à mediana e 101 com pontuação inferior à mediana; **IC 95\% OR = Intervalo de confiança de 95\% para a razão de chance; valor-p do teste de Wald estatisticamente significativo se p<0,05.

\section{DISCUSSÃO}

Realizamos um estudo tipo linha de base e seguimento investigando associações entre os significados atribuídos por idosos ao conceito "ser feliz na velhice" e as pontuações por eles obtidas em uma medida de qualidade de vida percebida (CASP-19). Idosos que não mencionaram nem saúde e funcionalidade e nem recursos materiais na linha de base tiveram maior probabilidade de pontuar alto na escala de qualidade de vida percebida no seguimento, do que aqueles que mencionaram esses significados. Os que citaram relações interpessoais tiveram maior probabilidade de pontuar alto na CASP-19, do que os que não os citaram. Participantes que pontuaram alto no fator autorrealização e prazer apresentaramse com maior probabilidade de mencionar suporte social e de não mencionar saúde e funcionalidade e recursos materiais. Foram observadas associações entre pontuação elevada no fator autorrealização e prazer, ausência de menções a saúde e funcionalidade e a recursos materiais, presença de menções a suporte social, e idade de 80 a 84 anos.

Os idosos manifestaram uma compreensão do conceito de felicidade na velhice como uma condição que apresenta aspectos hedônicos (ligados à satisfação das necessidades, à busca do prazer e à 
evitação do sofrimento) e eudaimônico (ligados à busca da excelência pessoal). Mostraram, também, que valorizam mais os aspectos eudaimônicos do que os aspectos hedônicos das experiências de felicidade e de qualidade de vida percebida e que percebem a saúde, a funcionalidade e os recursos materiais como aspectos do bem-estar na velhice, não como sua única ou mais crítica faceta, como comumente se pensa que os idosos pensam.

Pessoas em idade avançada que se diferenciam por desfrutar de boa saúde física e cognitiva para a idade, costumam supervalorizar a sua condição, quer porque, de fato, elas estão nos estratos superiores de adaptação do seu grupo de idade, quer porque se sentirem superiores aos iguais, em matéria de saúde e funcionalidade, cumpre objetivos compensatórios para a sua experiência de envelhecimento. Nas idades avançadas, ganham proeminência as experiências de bem-estar eudaimônico refletido em bem-estar psicológico, autodesenvolvimento e espiritualidade, bem como tornam-se cada vez mais importantes as relações familiares e de amizade e o apoio social, em face do inevitável declínio físico, cognitivo e social que acompanha o envelhecimento. Essas noções sobre os dados estão de acordo com a literatura teórica ${ }^{1,12,15,32,33}$ e com pesquisas sobre felicidade $^{2,4,6,8,9,11}$, qualidade de vida percebida ${ }^{23,24} \mathrm{e}$ bem-estar ${ }^{2,5,10,13}$. Neste estudo, a importância dada às relações interpessoais terá sido fortalecida pela forte presença feminina na amostra, dados que se repetem em pesquisas com idosos mais velhos, em diferentes países ${ }^{8,9,12,15-17,32}$. Uma outra particularidade deste estudo terá sido o realce angariado pelo fator senso de autorrealização e prazer e a ênfase atribuída pelos idosos ao autodesenvolvimento, reforçando a impressão de superioridade dos motivos eudaimônicos sobre os hedônicos na determinação do ajustamento dos idosos, replicando dados da pesquisa internacional ${ }^{12,17,19,33,34}$.

Circunstâncias do delineamento do Estudo Fibra, contribuíram para a diferenciação da amostra, em comparação com a de outros estudos de base populacional: nenhum participante apresentava deficit cognitivo sugestivo de demência e todos responderam à totalidade dos itens de dois instrumentos complexos, o que sugere que eram mais saudáveis do que os excluídos por esses critérios. Os que faleceram entre a linha de base e o seguimento (37,5\% das perdas) eram provavelmente mais frágeis e doentes do que os sobreviventes. É razoável supor que a não-localização de boa parte dos participantes da linha de base deveu essa condição ao fato de terem se mudado para a casa de filhos ou para instituições de longa permanência para idosos (ILPI), por doença, incapacidade ou viuvez.

Dessa forma, pode ter ocorrido um viés decorrente da sobrevivência dos participantes de condições de saúde mais robustas. Porém, mesmo aceitando essa hipótese, não deve ser descartada a possibilidade de a amostra abrigar considerável heterogeneidade dentro de cada grupo de idade. Com base em análises de grandes bancos de dados oficiais dos Estados Unidos e do banco de uma das ondas do Health and Retirement Study, Lowski et al. ${ }^{34}$ relataram que $48 \%$ da amostra de 51 a 54 anos, $42 \%$ dos de 65 a $69,38 \%$ dos de 70 aos $75,30 \%$ dos de 80 a 84 e $28 \%$ daqueles de 85 anos e mais classificaram a própria saúde como excelente e muito boa. Entre os de 51 e 54 anos, 96\% eram independentes para todas as AIVD (Atividades Instrumentais de Vida Diária) e ABVD (Atividades Básicas de Vida Diária), taxa que foi para $79 \%$ entre 80 e 84 anos e para $56 \%$ entre os de 85 anos e mais. O percentual dos diagnosticados como portadores de qualquer uma das cinco doenças crônicas mais prevalentes nos EUA foram $75 \%$ entre 51 e 54 anos, $50 \%$ entre 65 e 69 , e $35 \%$ entre os 80 e os $84^{34}$.

Note-se que a amostra foi majoritariamente feminina, o que terá contribuído para a maior frequência de menções aos temas relações interpessoais, relações familiares e bem-estar psicológico do que aos temas condições materiais e satisfação e prazer, ao passo que os homens tenderam a sobrevalorizar recursos materiais e saúde e funcionalidade. Trata-se de uma tendência observada em estudos similares ${ }^{8,9,12,16,18,20}$, devido a fatores genético-biológicos, socioeconômicos e de gênero.

Se por um lado as características da amostra desestimulam fazer amplas generalizações, por outro elas criaram condições para a observação de indivíduos septuagenários e octogenários que apresentavam padrões de funcionamento compatíveis com os de velhice ótima ou bem-sucedida dos pontos 
de vista biomédico e psicológico. Suas respostas nos dois tempos de medida refletem valores e significados afetivos e cognitivos socialmente compartilhados sobre felicidade e qualidade de vida na velhice, em interação com suas condições de vida e a maneira como viveram e estão vivendo a sua velhice. Não raciocinamos como se os significados observados na linha de base pudessem ser considerados como causas das pontuações em qualidade de vida percebida avaliada no seguimento. Tampouco planejamos o hiato de nove anos entre as duas medidas, mas planejamos servir-nos dele para compreender melhor as interações entre o significado de conceitos sociais complexos e medidas subjetivas de qualidade de vida na velhice avançada.

\section{CONCLUSÕES}

Este estudo tipo linha de base e seguimento oferece dados sobre aspectos relevantes da experiência de envelhecimento de idosos septuagenários e octogenários, especificamente sobre as associações sobre qualidade de vida referenciada por critérios psicológicos e as concepções dos idosos sobre felicidade na velhice. Revelou que há associações

\section{REFERÊNCIAS}

1. Steptoe A. Investing in happiness: the gerontological perspective. Gerontology. 2019;65:634-9. Disponível em: https://doi.org/ 10.1159/000501124 .

2. Luz MMC, Amatuzzi MM. Vivências de felicidade de pessoas idosas. Estud Psicol. 2008;25(2):303-7. Disponível em: http://dx.doi.org/10.1590/S0103166X2008000200014.

3. Rosa FHM, Cupertino APFB, Neri AL. Significados de velhice saudável e avaliações subjetivas de saúde e suporte social entre idosos recrutados na comunidade. Geriatr Gerontol Aging. 2009;3(2):62-9.

4. Camargo BV, Justo AM, Lima LMG, Rabelo DF, Neri A. Representações sociais de felicidade. In: Neri A, Guariento ME. Fragilidade, saúde e bem-estar em idosos Dados do Estudo Fibra Campinas. Campinas: Alínea; 2011. p. 239-56. entre os dois conjuntos de dados, entre os quais as mais importantes são aquelas presididas por questões de saúde e funcionalidade, bem-estar econômico, bemestar psicológico e aspectos hedônicos e eudaimônicos, estes mais valorizados pelos idosos do que aqueles.

Os dados estimulam a valorização do uso das abordagens qualitativas na pesquisa sobre felicidade, qualidade de vida percebida e correlatos. Igualmente, estimula a derivação de instrumentos úteis às práticas clínica, social e educacional, e à elaboração de políticas públicas centradas em concepções positivas sobre o envelhecimento. De natureza modificável, estas podem ser alvo de pesquisas e intervenções teórica e socialmente relevantes.

Editado: Maria Helena Rodrigues Galvão

\section{RESPONSABILIDADES PELA AUTORIA}

AL Neri planejou e coordenou o estudo, D Assumpção colaborou com a análise de dados e CNA Valero e TFG Meira analisaram os dados e prepararam a primeira versão do manuscrito, que foi revisado por AL Neri e D Assumpção.

5. Mantovani EP, de Lucca SR, Neri AL. Associações entre significados de velhice e bem-estar subjetivo indicado por satisfação em idosos. Rev Bras Geriatr Gerontol. 2016;19(2):203-22. Disponível em: https:// doi.org/10.1590/1809-98232016019.150041.

6. Pichler NA, Scortegagna HM, Dametto J, Frizon DMS, Zancanaro MP, de Oliveira TC. Reflexões acerca da percepção dos idosos sobre a felicidade e dinheiro. Rev Bras Geriatr Gerontol. 2019;22(2):e180185. Disponível em: https://doi. org/10.1590/1981-22562019022.180185.

7. Neri AL, Borim FSA, Riberiro LHM, Rabelo DF, Melo DM, Pinto JM, et al. O que os idosos entendem por velhice saudável e por ser feliz na velhice? In: Neri AL. Fragilidade e qualidade de vida na velhice. Campinas: Alínea; 2013. p. 341-62. 
8. Aburto KH, Rioseco MM, Moyano-Díaz E. Concept of happiness in adults from low-income class. Paidéia. 2017;27:386-94. Disponível em: https://doi. org/10.1590/1982-432727s1201703 .

9. Puello-Alcocer EC, Ortega-Montes JE, ValenciaJiménez $\mathrm{N}$. The meaning of happiness from the perception of a group of older adults from the city of Montería (Córdoba, Colombia). Index Enferm. 2018;27(1-2):33-7.

10. Diener E, Oishi S, Tay L. Advances in subjective well-being research. Nat Hum Behav. 2018;2(4):25360. Disponível em: https://doi.org/10.1038/s41562018-0307-6.

11. Ryan RM, Deci EL. On happiness and human potentials: a review of research on hedonic and eudaimonic well-being. Ann Rev Psychol. 2001.52:141-66. Disponível em: https://doi. org/10.1146/annurev.psych.52.1.141.

12. Komatsu H, Yagasaki K, Kida H, Eguchi Y, Niimura H. Preparing for a paradigm shift in aging populations: listen to the oldest old. Int J Qual Stud Health Well-being. 2018;13(1):e1511768. Disponível em: https://doi.org/ 10.1080/17482631.2018.1511768.

13. Carmel S. Health and well-being in late life: gender differences worldwide. Front Med. 2019;6(218):1-

11. Disponível em: https://doi.org/10.3389/ fmed.2019.00218.

14. Ryff CD. Well-being with soul: science in pursuit of human potential. Perspect Psychol Sci. 2018;3(2):242-8. Disponível em: https://doi. org/10.1177/1745691617699836.

15. Aguilera-Velasco MA, Acosta-Fernández M, FrancoChávez SA, Soltero-Avelar R. Cultural consensus about happiness among the elderly. Eur Scient J. 2017;13(20):1-9. Disponível em: https://doi. org/10.19044/esj.2017.v13n20p55.

16. Manasatchakun P, Chotiga P, Roxberg A, Asp M. Healthy ageing in Isan-Thai culture-a phenomenographic study based on older persons' lived experiences. Int J Qual Stud Health Well-being. 2016;11:1-9. Disponível em: http://doi.org/10.3402/ qhw.v11.29463.

17. Doh YY, Chung JB. What types of happiness do Korean adults pursue? : comparison of seven happiness types. Int J Environ Res Public Health. 2020;17(5):1-10. Disponível em: https://doi.org/ 0.3390/ijerph17051502.

18. Hernandez R, Carnethon M, Penedo FJ, Martinez L, Boehm J, Schueller SM. Exploring well-being among US Hispanics/Latinos in a church-based institution: a qualitative study. J Posit Psychol. 2016;11(5):511-21. Disponível em: https://doi.org/ 10.1080/17439760.2015.1117132.
19. Russo-Netzer P, Littman-Ovadia H. "Something to live for": experiences, resources, and personal strengths in late adulthood. Front Psychol. 2019;10:e2452. Disponível em: https://doi.org/ 10.3389/fpsyg.2019.02452.

20. Browning CJ, Qiu Z, Yang H, Zhang T, Thomas SA. Food, eating, and happy aging: the perceptions of older Chinese people. Front Public Health. 2019;7:1-12. Disponível em: https://doi.org/ 10.3389/ fpubh.2019.00073.

21. Varin M, Palladino E, Lary T, Baker M. An update on positive mental health among adults in Canada. Health Promot Chronic Dis Prev Canada. 2020;40(3):86-91. Disponível em: https//:doi. org/10.24095/hpcdp.40.3.04.

22. Boggartz T. Quality of life in old age-a concept analysis. Int J Older People Nurs. 2015;11(1):55-69. Disponível em: https://doi.org/ 10.1111/opn.12089.

23. van Leeuwen KM, van Loon MS, van Nes FA, Bosmans JE, de Vet HCW, Ket JCF, et al. What does quality of life mean to older adults? A thematic synthesis. PLoS ONE. 2019;14(3): e0213263. Disponível em: https://doi.org/10.1371/journal.pone.0213263.

24. Hyde M, Wiggins RD, Higgs P, Blane DB. A measure of quality of life in early old age: The theory, development and properties of a needs satisfaction model (CASP-19). Aging Ment Health. 2003;7(3):186-94. Disponível em: https://doi.org/ 10.1080/1360786031000101157.

25. Neri AL, Borim FSA, Batistoni SST, Cachioni M, Rabelo DF, Fontes AP, et al. Nova validação semântico-cultural e estudo psicométrico da CASP-19 em adultos e idosos brasileiros. Cad Saúde Pública. 2018;34(10):e00181417. Disponível em: https://doi. org/ 10.1590/0102-311x00181417.

26. Neri AL, Yassuda MS, de Araújo LF, Eulálio MC, Cabral BE, de Siqueira MEC, et al. Metodologia e perfil sociodemográfico, cognitivo e de fragilidade de idosos comunitários de sete cidades brasileiras: estudo FIBRA. Cad Saúde Pública. 2013;29(4):77892. Disponível em: https://doi.org/ 10.1590/S0102311X2013000400015.

27. Neri AL, Yassuda M. Características sociodemográficas dos participantes e procedimentos do Fibra 80+. In: Neri AL, Borim F, Assumpção D. Octogenários em Campinas: dados do FIBRA 80+. Campinas: Alinea; 2019. p.21-38.

28. Bertolucci PHF, Brucki SMD, Campacci SR, Juliano Y. O mini-exame do estado mental em uma população geral: impacto da escolaridade. Arq Neuropsiquiatr. 1994;52(1):1-7. Disponível em: https://doi.org/ 10.1590/S0004-282X1994000100001. 
29. Brucki SMD, Nitrini R, Caramelli P, Bertolucci PHF, Okamoto IH. Sugestões para o uso do mini-exame do estado mental no Brasil. Arq Neuropsiquiatr . 2003;61(3B):777-81. Disponível em: https://doi.org/ 10.1590/S0004-282X2003000500014.

30. Bardin L. Análise de conteúdo. Lisboa: Edições 70; 2006.

31. Iphofen R, Tolich M. The SAGE handbook of qualitative ethics. research London: SAGE Publications; 2018.
32. Ryff CD, Keyes CLM. The structure of psychological well-being revisited. J Pers Soc Psychol.1995;69(4):719-27. Disponível em: https://doi. org/10.1037//0022-3514.69.4.719.

33. Lang FR, Carstensen LL. Time counts: future time perspective, goals, and social relationships. Psychol Aging. 2002 17(1):125-39. Disponível em: https://doi. org/10.1037/0882-7974.17.1.125.

34. Lowsky D, Olshansky J, Bhattacharya J, Goldman DP. Heterogeneity in healthy aging. J Gerontol Ser A Biol Sci Med Sci. 2014;69(6):640-9. Disponível em: http://doi.org/:10.1093/gerona/glt162. 